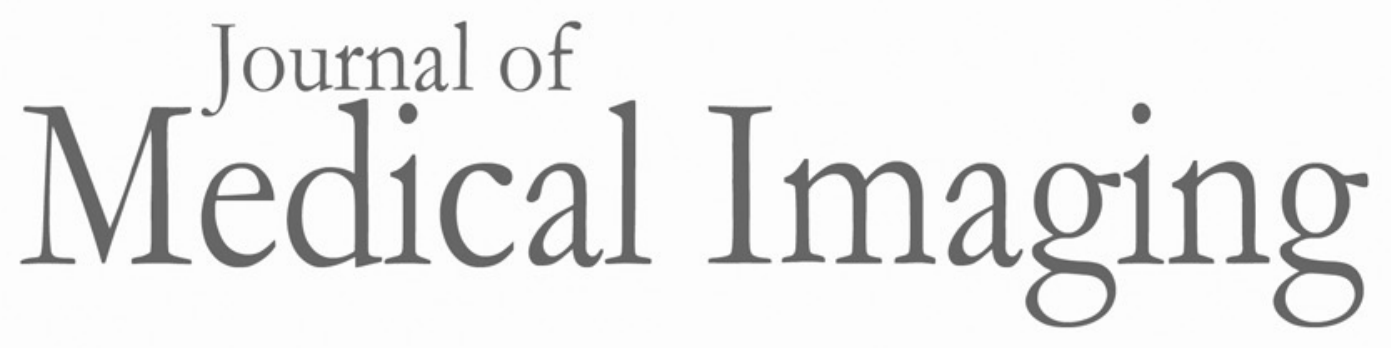

Medicallmaging.SPIEDigitalLibrary.org

\title{
JMI 2018 List of Reviewers
}


The Journal of Medical Imaging would like to sincerely thank the following individuals who served as reviewers in 2018 . The success of our publication hinges on the voluntary contributions of time and energy put forth by these professionals.

\begin{tabular}{|c|c|c|}
\hline Craig Abbey & Ciprian Catana & Nils Gessert \\
\hline Ehsan Adeli & Oriol Caudevila & Olivier Gevaert \\
\hline Carla Agurto Rios & Kenny Cha & Michael Ghaly \\
\hline M. Faizal Ahmad Fauzi & Kish Chakrabarti & Zahra Ghanian \\
\hline Rifat Ahmed & Heang-Ping Chan & Zeinab Ghassabi \\
\hline Walter Akers & Arion Chatziioannou & Nooshin Ghavami \\
\hline Mohammad A. Al-Masni & Buxin Chen & Eli Gibson \\
\hline Adam Alessio & Elvis Chen & Howard Gifford \\
\hline Sharib Ali & Liyuan Chen & Hannah Gilmore \\
\hline Mehdi Alilou & Min Chen & Stephen Glick \\
\hline Robert Alvarez & Shuqing Chen & Tsutomu Gomi \\
\hline Guy Amit & Weijie Chen & Christian Graff \\
\hline Emran M. Abu Anas & Xinjian Chen & Albert Gubern Merida \\
\hline Mustafa Umit Arabul & Gong Cheng & Junfeng Guo \\
\hline Jose Luis Arce-Diego & Ruida Cheng & Ranu Gupta \\
\hline Samuel Armato & Matthew Man Hin Cheung & Lubomir Hadjiiski \\
\hline Susan Astley & Teodora Chitiboi & Dieter Haemmerich \\
\hline Albert Attia & Seungryong Cho & Shizhong Han \\
\hline William Auffermann & Darin Clark & Per Christian Hansen \\
\hline Ricardo Avila & Eric Clarkson & Takeshi Hara \\
\hline Suyash Awate & Guy Cloutier & Charles Hatt \\
\hline Paulo Azevedo-Marques & Jean-Pierre Da Costa & Mathieu Hatt \\
\hline Cristian Badea & Jeremy Dahl & Xiaoyuan $\mathrm{He}$ \\
\hline Ulas Bagci & Mini Das & Xin $\mathrm{He}$ \\
\hline Predrag Bakic & Niti Dhutia & Karl-Heinz Herrmann \\
\hline Andriy Bandos & Solomon Diamond & Stephen Hillis \\
\hline Dean Barratt & Trushali Doshi & Yasushi Hirano \\
\hline Bruno Barufaldi & Qi Dou & Kenneth Hoffmann \\
\hline Mark Basham & Jason Dowling & David Holmes, III \\
\hline Khayrul Bashar & Trafton Drew & Michal Hradis \\
\hline Magnus Bath & Karen Drukker & Jiang Hsieh \\
\hline Anton Becker & Jean Dumoulin & Scott Hsieh \\
\hline Niha Beig & Jan Ehrhardt & Houchun $\mathrm{Hu}$ \\
\hline Amira Ben Rabeh & Babak Ehteshami Bejnordi & Sijung $\mathrm{Hu}$ \\
\hline Benjamin Berkels & Hany Elsalamony & Lin Huang \\
\hline Michael Berks & Marco Endrizzi & Henkjan Huisman \\
\hline Jorn Bersvendsen & Avinash Eranki & Johan Hulleman \\
\hline Sanjoy Bhattacharya & Stephanie Eyerly-Webb & Andreas Husch \\
\hline Junguo Bian & Rebecca Fahrig & Juan Eugenio Iglesias \\
\hline Mustafa Bicer & Claas Falldorf & Lynda Ikejimba \\
\hline Hamidullah Binol & Andriy Fedorov & Michael Insana \\
\hline Kristina Bliznakova & Aaron Fenster & Ciprian Ionita \\
\hline Sebastian Bodenstedt & Martin Fergie & Hayato Itoh \\
\hline Hans Bornefalk & James Ford & Anna Jerebko \\
\hline Hilde Bosmans & Augustus Fountain III & Jingfeng Jiang \\
\hline Nick Bottenus & Sean Foxley & Dakai Jin \\
\hline Jelena Bozek & Alfred Franz & Yueming Jin \\
\hline Jovan Brankov & Erik Fredenberg & Amod Jog \\
\hline Dag Breiby & Steve Freeman & Paul Johns \\
\hline Paul Bromiley & Nagarajan Ganapathy & Marc Kachelriess \\
\hline Adam Budde & Ziba Gandomkar & Samuel Kadoury \\
\hline Lindsay Busby & Aimilia Gastounioti & Raheleh Kafieh \\
\hline Brett Byram & Marios Gavrielides & J. Kalpathy-Cramer \\
\hline Weidong Cai & Jiajia $G e$ & Andrew Karellas \\
\hline Enrico Caiani & Baris Gecer & Marek Karolczak \\
\hline Jochen Cammin & Marcel Gehrung & Nico Karssemeijer \\
\hline W. Scott Campbell & Yasmeen George & Ian Katz \\
\hline Ann-Katherine Carton & Arkadiusz Gertych & Brad Keller \\
\hline
\end{tabular}


Mawia Khairalseed

Fahmi Khalifa

John Kipritidis

Takayuki Kitasaka

A. Kizhakke Puliyakote

Stefan Klein

Thomas Koenig

Dimitri Komatitsch

Robert Korez

Cemal Kose

Kivanc Kose

Aikaterini Kotrotsou

Ganapathy Krishnamurthi

Elizabeth Krupinski

Hanif Ladak

Kemi Ladeji-Osias

Guenter Lauritsch

Sergej Lebedev

Junghoon Lee

Nikolas Lessmann

Chiye $\mathrm{Li}$

Hongjun Li

Hui Li

Jiao Li

$\mathrm{Li} \mathrm{Li}$

Yu Lin

Cristian Linte

Damien Litchfield

Stephen Littlefair

Jiamin Liu

Mengyang Liu

Xinyang Liu

Yilin Liu

Yun Liu

Cheng Lu

Yihuan Lu

Kevin Ma

Alistair Mackenzie

Ilias Maglogiannis

Faisal Mahmood

Harish Malhotra

Steve Mann

Oge Marques

Anthony Maxwell

Matthew McGarry

Milica Medved

Carlos Mello

Claudia Mello-Thoms

Thomas Mertelmeier

Mohammad Badrul Miah

Sara Moccia

Dimple Modgil

Pim Moeskops

James Monaco

Amirreza Moradi

Mehdi Moradi

Pedro Morais

Antonio Moreira

Bert Mueller

Karthik Muthuswamy
Sreyankar Nandy

Rohit Nayak

Hamid Neshat

Muhammad K. Khan Niazi

Bo Ning

Humaira Nisar

loan Notingher

Matthew Nyflot

Christine O'Brien

Nancy Obuchowski

Hirohisa Oda

Masahiro Oda

Idowu Okuwobi

Rudolf Oldenbourg

Rahul Pachauri

Anastasia Pampouchidou

Tinsu Pan

Kevin Parker

Bahram Parvin

Rahul Paul

Joao Pedrosa

Raquel Perez Lopez

Mailyn Perez-Liva

Mats Persson

Jens Petersen

Amir Pourmorteza

Pottumarthi Prasad

Niladri Puhan

Jin Qi

Wei Qian

Hossein Rabbani

K. Rajamanickam

Senthil Ramamurthy

Raj Ratwani

Geetha Rayarao

Marcel Reginatto

Ingrid Reiser

Samuel Remedios

Liqiang Ren

Stefano Ricci

Taylor Richards

Leticia Rittner

Alejandro Rodriguez

Georg Rose

Sean Rose

Holger Roth

Laurence Rouet

Francois Rousseau

Mirabela Rusu

Suresh Sadananthan

Olivier Salvado

Ravi Samala

Frank Samuelson

Manojkumar Saranathan

Pinaki Sarder

Alexander Schlaefer

Taly Schmidt

Alexander Seitel

Anando Sen

Caglar Senaras
Olga Senyukova

M. Shafiq ul Hassan

Wei Shao

Jennifer Shell

John A. Shepherd

Chun-Chien (Andy) Shieh

Emil Sidky

Arkadiusz Sitek

Orjan Smedby

Justin Solomon

Hamid Soltanian-Zadeh

Chaolong Song

Wenwei Song

Jacob Sosna

Rachel Sparks

Yeshwanth Srinivasan

Roman Starosolski

Darko Stern

Martin Stumpe

Nagesh Subbanna

Radhakrishnan Sudhakar

Ronald Summers

Timothy Szczykutowicz

Raphael Sznitman

Fuk Hay Tang

Xiangyang Tang

Jesse Tanguay

Kai Thomenius

Sara Thrower

Zhiqiang Tian

Anders Tingberg

Pallavi Tiwari

John Tomaszewski

Turid Torheim

Dragos Trinca

Chun-Ming Tsai

Hsin-Wu Tseng

Simona Turco

Ismael Baris Turkbey

Timothy Turkington

Nicholas Tustison

Christopher Uff

James Uhlir

Alireza Vard

Francois Varray

Harini Veeraraghavan

Dmitry Vengertsev

Satish Viswanath

Guobao Wang

Hesheng Wang

Hui Wang

Shang Wang

Xin Wang

Xiong Wang

Andreas Weinmann

William Wells

Gezheng Wen

Heather Whitney

Lauren Williams

David Wilson 
Joshua Wilson

William Winfree

Michael Winkler

Teresa Wu

Adam Wunderlich

Wenfeng Xia

Lei Xing

Kaikai Xu

Yiwen Xu

Ziyue $\mathrm{Xu}$

Ke Yan

Pingkun Yan
Dong Yang

Qingsong Yang

Wanneng Yang

Thomas Yankeelov

Brian Yanoff

Bulent Yener

Xin Yin

Pengxiao Zang

Amin Zarshenas

Wojciech Zbijewski

Berit Zeller-Plumhoff

Haichong Zhang
Ran Zhang

Sheng Jun Zhang

Xuezhu Zhang

Zheng Zhang

Wei Zhao

Yanyu Zhao

Yushan Zheng

Mu Zhou

Hancan Zhu

Jie Zhu

Maria Zuluaga

Alexander Zwanenburg 